\title{
THE FRACTIONAL METHOD OF BLOOD PRESSURE DETERMINATION-A CONTRIBUTION TO THE STUDY OF BLOOD PRESSURE IN CARDIAC ARRHYTHMIAS *
}

\author{
EUGENE S. KILGORE \\ SAN FRANCISCO
}

Among the sources of error in human blood pressure measurements an important factor, which has received comparatively little attention, is variation in pressure between individual pulse waves or between groups of them. It is well known that undulations in systolic and diastolic pressure occur commonly in normal persons with each respiration or at somewhat longer intervals; and among the cases of cardiac arrhythmia, there may be the widest pressure differences even between consecutive pulse waves. The custom of clinicians to accept the average or the highest of several determinations, or simply the first reading made, while tolerably meeting the needs of practice among cases with regular heart action, amounts to the roughest kind of guess-work in cases of marked arrhythmia. Mackenzie ${ }^{1}$ gave up the attempt to measure blood in cases of auricular fibrillation. It will be shown, however, that notwithstanding the great differences between individual waves in these cases, the average pressure from minute to minute may be remarkably stable; and that the determinations, though not expressible as single figures, may have as much accuracy as the ordinary clinical methods will yield in normal cases.

It will be unnecessary here to discuss the numerous attempts which have been made to obtain graphic records showing quantitatively the rapid changes in human arterial pressure. Suffice it to say that up to the present the sphygmographs and plethysmographs used as receivers have been beset with technical difficulties which make them not only inconvenient, but uncertain in their results. ${ }^{2}$

Another group of endeavors has had more limited aims, such as:

a. To show the highest and lowest systolic pressures in a series of irregular beats, by noting the cuff pressure at which no beats come through and that at which all come through. ${ }^{3}$

* From the Department of Medicine, University of California.

1. Mackenzie: Heart, 1911, ii, 283.

2. Weber: Arch. f. Anat. u. Physiol., 1913, Physiol. Abt.

3. Wybauw: Ztschr. f. klin. Med., 1911, 1xxiii, 214. 
b. To show qualitatively variations above or below a known systolic pressure by noting the appearance and disappearance of the "change in form" in an Erlanger sphygmomanometer curve. ${ }^{4}$

c. By the method of intermittent escapement and the use of the graphic oscillatory criteria, to show the general level of systolic and diastolic pressures when there is variation among the beats. ${ }^{5}$

d. To compare the number of beats coming through one radial artery with those coming through the other when varying degrees of pneumatic pressure are applied to one arm, using a sphygmograph on each wrist for registering the beats. ${ }^{6}$

Observations similar to the last mentioned were made by James and Hart, ${ }^{7}$ but without the use of instruments other than the ordinary clinical blood pressure apparatus. Their method was to count the heart rate by auscultation of the precordia, and by palpation of the radial artery the rate without arm compression and also with different degrees of compression by the pneumatic cuff. From these observations they could state approximately: (1) The number of beats per minute which fail to reach the periphery as palpable waves (the so-called "pulse deficit"), and (2) the number per minute which have a systolic pressure of $140 \mathrm{~mm}$. $\mathrm{Hg}, 130,120$, and so on. ${ }^{8}$ The pressure variation among the pulse waves in the arm they designate the "relative deficit." What they call the average pressure is obtained by multiplying, for example, by 140 the number of beats per minute which they considered to have that pressure, by 130 the number with that pressure, and so on; then adding all these products and dividing the sum by the heart rate as counted at the apex. They did not apply the procedure to diastolic pressures.

A simple method such as this is attractive on account of the nonrequirement of machinery other than the ordinary blood pressure apparatus. The only advantages which could be claimed for one of the graphic methods would be the doubtful one of increased accuracy in identifying and counting the waves which passed under the compressing cuff, and the possibility of registering simultaneously the rate of the unobstructed pulse. The pulse rate would, of course, be needed for correct interpretation of the results if it changed materially from minute to minute. That such is not the case, however, in cases of auricular fibrillation, where this method finds its greatest usefulness, is the experience of James and Hart and of myself. Moreover, in the

4. Erlanger and Festerling: Jour. Exper. Med., 1912, xv, 370.

5. Erlanger: Johns Hopkins Hosp. Rep., 1904, xii, 53.

6. Silberberg: Brit. Med. Jour., April 6, 1912.

7. James and Hart: Am. Jour. Med. Sc., January, 1914, p. 63.

8. A slight error in these calculations will be referred to later. 
method of plotting the results here proposed, any noteworthy change of the pulse rate or of the general run of pressure during the experiment would at once become evident as a distortion of the curve. The essential "smoothness" of such a curve is evidence of the constancy of the circulatory conditions during the experiment.

A method differing from that of James and Hart in two important particulars (the interpretation of systolic determinations and the inclusion of diastolic readings) was initiated in the University of California Hospital about one year before their publication, and the illustrations herewith presented are taken from the hospital records of the last two years. We have designated it the fractional method because of its obvious similarity to the fractional procedures of chemistry, bacteriology, etc. It will best be described by examples:

Section I.-Cases in which the Lowest Systolic Pressures arc Above the Highest Diastolic Pressures. Medical Case 8243, admitted Nov. 13, 1914; diagnosis, mitral stenosis, auricular fibrillation. Although the heart was absolutely irregular, practically every beat had a corresponding radial pulse. With circular compression of 150 $\mathrm{mm}$. $\mathrm{Hg}$ applied by a $12 \mathrm{~cm}$. pneumatic cuff to the upper arm, no wave: were felt in the radial artery of the same side during half a minute. After emptying the cuff and resting the arm a few seconds, the pressure was again raised and held for one half minute at $145 \mathrm{~mm}$. $\mathrm{Hg}$. During this time two waves were felt at the wrist. Then there vas another rest of the arm, inflation of the cuff, and half minute count of the waves which were able to lift $140 \mathrm{~mm}$. $\mathrm{Hg}$, and so on down to $110 \mathrm{~mm}$. $\mathrm{Hg}$, which obstruction all the waves were able to pass.

For the fractional determination of diastolic pressure a stethoscope was placed over the artery a few centimeters below the cuff, and with further similar decrements of cuff pressure, the numbers of arterial sounds heard in half minute intervals were counted (i. e., the "ffifth phase" or sound disappearance criterion was followed).

The results are shown graphically in Figure 1, in which the dots represent the rate per minute (indicated by the corresponding figures on the base line) of waves which pass under a given degree of pressure in the pneumatic cuff (indicated by the corresponding figures at the left), and where the circles in a similar way indicate the counts of sounds. The connecting lines, it will be noted, are drawn with the purpose of representing the probable truth rather than with absolute faithfulness to the dots and circles: i. e., small irregularities have been "smoothed out." This undoubtedly increases the accuracy of the lines (provided of course that enough counts have been made to bring the

9. Gittings: The Archives Int. Med., 1910, vi, 196. 
dots and circles approximately in line), since it is what happens wher: such curves are based on much larger numbers of counts. The purpose of this paper being primarily to describe the method, in the interest of clearness and simplicity in the figures which follow only the smoothed out lines are shown.

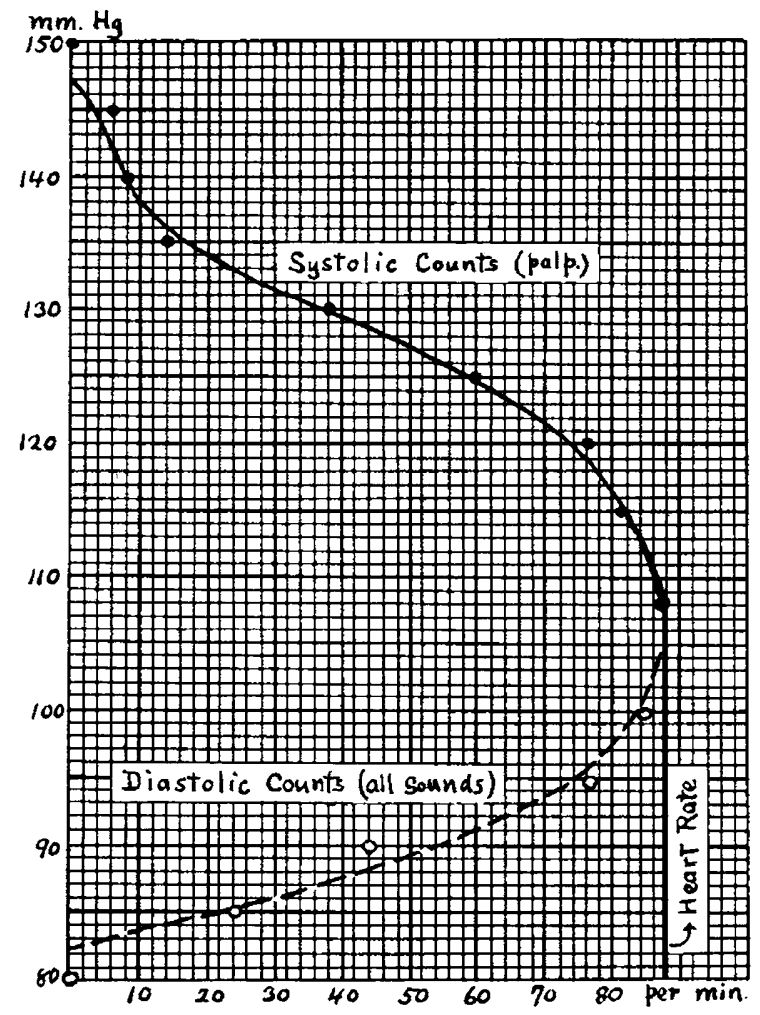

Fig. 1.-Showing the relation of systolic and diastolic pressures in a typical case of auricular fibrillation in which the diastolic pressure of one cycle never exceeds the systolic pressure of another. In this as well as the following figures the numbers at the left indicate millimeters of mercury pressure in the pneumatic cuff; those at the bottom the rate per minute of beats counted in various ways. The upper line here represents the results of palpatory systolic counts, the lower line fifth phase diastolic counts.

Figure 1 may be better understood if it is imagined that the 88 pulse waves in a minute are represented by sticks with lengths proportional to the systolic pressures, and that these sticks are stood up in the order of their lengths, the longest on the left and the shortest on the right. Their tops would form the upper curve in the figure. A similar set of sticks representing diastolic pressure would form the lower curve if the longer ones were placed on the right. 
It will be seen that the systolic pressure varies between 110 and $145 \mathrm{~mm} . \mathrm{Hg}$, and that its average is in the neighborhood of 125 to 130 . To continue the illustration of the sticks, the average systolic pressure would be represented by their average length, which would be obtaired by dividing the sum of their lengths by their number. The average length of 88 equally spaced ordinates dropped from the systolic curve in Figure 1 is about 129.

An approximately correct average can be obtained with less labor by dealing with groups rather than points on the line representing single waves. For example, according to the figure, there were six waves per minute with a pressure between 145 and 150 . These six may all be considered to have a pressure half way between the two limits: i. e., $147.5 \mathrm{~mm}$. $\mathrm{Hg}$. In this way the complete calculation would be as follows:

(a) (b) (c)*

$6-0=6 . \quad 6 \times 147.5=885$

$8-6=2 . \quad 2 \times 142.5=285$

$14-8=6 . \quad 6 \times 137.5=825$

$38-14=24 . \quad 24 \times 132.5=3,180$

$60-38=22 . \quad 22 \times 127.5=2,805$

$76-60=16 . \quad 16 \times 122.5=1,960$

$82-76=6 . \quad 6 \times 117.5=705$

$88-82=6 . \quad 6 \times 112.5=675$

$$
\overline{11,320} \div 88=128.6=\text { average systolic pressure. }
$$

* In the figures shown herewith the numbers on the base line correspond to the figures in column $a$ in the above tabulation; i. e., they represent total numbers of waves felt or sounds heard at the corresponding cuff pressures. For some purposes it would be an advantage to construct curves in which the base line figures represented the quantities in column $c$, i. e., the number of beats which have a certain pressure. In such a figure taken from a normal case there would be two narrow curves separated by the amount of the pulse pressure, while if it were from a very irregular case the diastolic and systolic curves might overlap. The work of constructing these additional curves is unnecessary if it is remembered that their lateral extent would depend on the slope of the curves here employed. In the figures herewith, the more uniform the pressure of the beats the more nearly horizontal the lines, and the more variable the pressure the more sloping the lines.

It will be noted that this average differs by only about one-half millimeter from that obtained by measuring the whole 88 ordinates. In the averages calculated by James and $\mathrm{Hart}^{7}$ only half as many multiplications were made, i. e., waves were considered together which were within limits $10 \mathrm{~mm}$. $\mathrm{Hg}$ apart instead of $5 \mathrm{~mm}$. Undoubtedly the additional error introduced in this way is still well within the limits of accuracy of the method as a whole. A mistake of approximately $5 \mathrm{~mm}$. Hg, however, appears throughout their figures, which is unnecessary and easily corrected. For example, a group of beats which ranged in pressure from 130 to $140 \mathrm{~mm}$. $\mathrm{Hg}$ they counted as 
having a pressure of 130 . On the chances, the average pressure of this group would be near the midpoint or 135 rather than the lower limit of 130 .

The diastolic pressure is calculated from the lower curve in Figure 1 in the same way as systolic pressure, thus:

(a) (b) $(c) *$

$24-0=24$.

$44-24=20$.

$76-44=32$.

$84-76=8$.

$24 \times 82.5=1,980$
$20 \times 87.5=1,750$
$32 \times 92.5=2,960$
$8 \times 97.5=780$
$4 \times 102.5=410$

$88-84=4$.

$\overline{7,880} \div 88=89.5=$ average diastolic pressure.

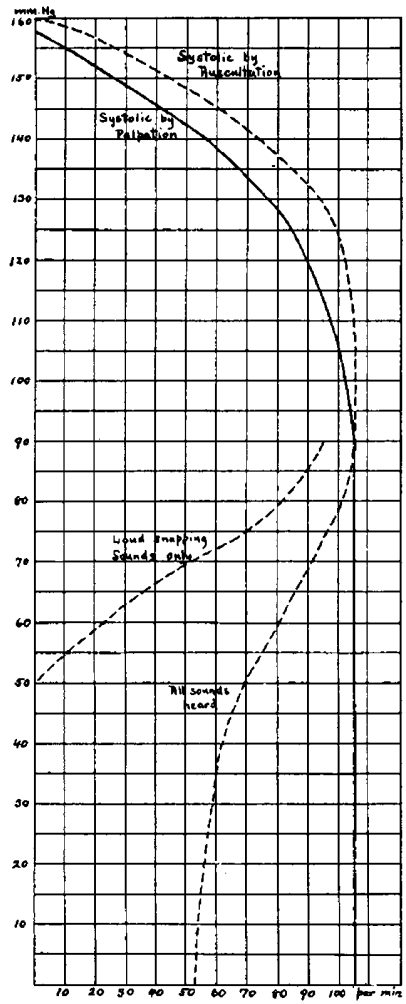

Fig. 2.-Showing the relation between palpatory (solid line) and auscultatory (broken line) systolic readings, and between fourth phase and fifth phase diastolic readings. From a case of hyperthyroidism and auricular fibrillation.

Obvious variations of the technic are to use the auscultatory method for systolic determinations and the "fourth phase" or sound transition index for diastolic readings; $i$. e., to count all the arterial sounds heard instead of the pulse waves felt in the systolic region, and in the diastolic region to include in the counts only the loud staccato notes and omit the dull distant ones. Figure 2 shows 
side by side the results obtained by the use of the palpatory and auscultatory criteria for systolic pressure, and of sound transition and sound disappearance for diastolic pressure. It will be seen that in these fractional determinations there is present the usual excess of auscultatory over palpatory systolic readings, and of "fourth phase" over "ffifth phase" diastolic readings. The case from which this was obtained was one of auricular fibrillation with hyperthyroidism. The pulse approached the Corrigan in type, which accounts for the persistence of many of the arterial sounds during little or no cuff pressure.

For purposes of comparison it is desirable to use the same criteria for the determinations in these cases of arrhythmia as in ordinary cases. For general use in systolic determinations our choice has been the palpatory criterion, on account of its convenience, and we believe, equal or greater reliability. ${ }^{10}$ For diastolic readings we ordinarily use whichever auscultatory criterion seems the clearer, and indicate in the record which is used. In fractional determinations the "fifth phase" criterion is usually preferable, as it relieves the observer of the necessity of differentiating between qualities of sounds, and also takes away the danger of confusing sounds above the "fourth phase" with those below it. (In the second and third phases the murmur is frequently indistinct and the sounds very dull and distant.) A few cases, such as aortic insufficiency, will have audible sounds over the arteries with little or no compression; these necessitate the use of the sound transition criterion. Unless otherwise stated it is understood that the remaining observations here referred to were all obtained by the use of the palpatory index for systolic pressure and the disappearance of sounds for the diastolic.

Section 2.-Cases in which the Diastolic Pressure of Some Beats Exceeds the Systolic Pressure of Others. Figure 3 (Case 9229, May 21, 1915) illustrates this condition. Instead of coinciding as in Figure 1, the lines representing, respectively, systolic and diastolic pressure intersect; i. e., apparently the diastolic pressure of some cycles exceeds the systolic pressure of others. When this is so, it is necessary to make certain allowances in interpreting the diastolic counts with the higher cuff pressures, for the reason that not all of the silent waves are so because their diastolic pressures are above that in the cuff; some are not heard because they fail entirely to pass the cuff. An example in Figure 3 is the count of sounds when the cuff pressure was $115 \mathrm{~mm}$. Hg. The figure shows that the rate of these sounds was 58 per minute, which would mean, according to the

10. Kilgore: A Quantitative Determination of the Personal Factor in Blood Pressure by the Auscultatory Method, The Archives Int. Med., this issue, p. 893. 
usual interpretation, that fifty-eight waves per minute had a diastolic pressure lower than $115 \mathrm{~mm}$. Hg. But from the palpatory systolic counts it is known that even less cuff pressure was able to hold back entirely some of the smaller waves (at least so far as the finger could determine), and therefore, wrongfully for our results, exclude them from the stethoscope counts. The upper end of the diastolic line, therefore, needs to be corrected by bending it toward the right.

To determine the amount of correction to be made in the diastolic line, it is first necessary to make some of the systolic readings by auscultation as well as by palpation and to correct the lower end of the auscultatory systolic line (where beats may be missed on account of high diastolic pressure as well as on account of low systolic pressure) in accordance with the palpatory readings; $i$. e., so that the vertical

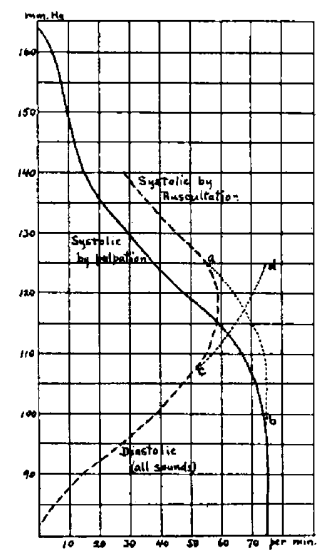

Fig. 3.-Showing the correction and extension of the upper end of the diastolic line in cases in which the diastolic pressure of some cycles exceeds the systolic pressure of others. Case of premature contractions followed by alternating pulse waves.

distance between the two lines shall be everywhere the same as at the higher pressure levels. ${ }^{11}$ The dotted portion $(a b)$ of the systolic auscultatory line in Figure 3 has been thus corrected. The difference between each point in this corrected auscultatory systolic line and the heart rate per minute, equals the number of waves per minute which are silent at that cuff pressure on account of their lower systolic pressures, and is therefore the number of beats to be added in order to correct the diastolic line at the corresponding pressure. For example,

11. It is assumed that the relation between palpatory and auscultatory systolic readings is about the same at different pressure levels. This probably is not strictly true, but it is certain that any error from this source is small and well within the limits of accuracy of the method as a whole. 
at pressure 110 this corrected auscultatory systolic line indicates 72 per minute, which is three short of the heart rate of 75 . Three beats are therefore added to the diastolic line at pressure 110 . Similarly at 115 , five beats are to be added, and so on. The dotted portion ( $c d$ ) of the diastolic line in Figure 3 was constructed in this way.

From these corrected lines the average systolic and diastolic pressures may be calculated as described in Section 1. The cases in which it is necessary thus to correct the diastolic line are comparatively few, as even in very marked cases of arrhythmia the diastolic line does not intersect the systolic line until both are at or near their limit. And when they cross only slightly before this, the diastolic line can be roughly corrected by "free hand exterpolation" within the limits of accuracy of the method.

Section 3.-Cases in which without Brachial Compression Some of the Heart Beats Fail to Reach the Radial Artery as Palpable Waves. Cases with abortive beats ${ }^{12}$ form a large proportion of the patients with absolute arrhythmia in the stage of decompensation, and a considerable number of patients with premature contractions. Clinically there is no method at present of measuring the exact pressure of these small beats, but they can be placed between certain rough limits. Those which produce both first and second heart sounds may be assumed to have opened the semilunar valves ${ }^{13}$ (presumably both pulmonic and aortic), and therefore to have exceeded the diastolic pressure of the preceding beat. Isolated first heart sounds, on the other hand, may be taken to indicate beats with systolic pressure below the existing diastolic pressure in the aorta.

Unfortunately the diastolic pressure as a limit is itself quite variable in most of these cases (in Figure 3, for example, it varies from 82 to $125 \mathrm{~mm}$. $\mathrm{Hg}$ ). Since, however, a prominent factor in causing

12. The difference between the apex and the radial counts has been referred to as the "pulse deficit." "Abortive beats" has seemed to us a better term because its meaning is clear and it directs attention to the heart, in respect to which these beats are important, rather than to the pulse, where they are not so important. Even in the presence of many abortive beats, there may be an ample number of large beats for the needs of the peripheral circulation; and from this point of view it seems not quite appropriate to speak of the small beats as a deficit. Indeed they might with less impropriety be referred to as a surplus.

13. Professor Joseph Erlanger has verbally called my attention to the possibility of second heart sounds occurring without a preliminary opening of the semilunar valves. It is quite conceivable that the systolic pressure on the side of the ventricle may at times be sufficient partially to relax the tension on the valves without opening them, and that their return to the tense position at the beginning of diastole may produce a sound. Whether or not this actually takes place would of course be difficult to prove; if it does, the sounds thus produced might be expected to be less intense than the second sounds of the effective beats. 
these small ventricular discharges (or attempted discharges) is insufficient ventricular filling, due to the shortness of the preceding diastole, and since it is usually the long pauses that bring about the low diastolic pressures, it may be inferred that these abortive beats usually happen during relatively high diastolic pressure. Occasionally, particularly in some cases of premature contractions, several small beats follow each other in rapid succession; the output of these beats may be negligible, and arterial pressure during the short interval may therefore decline rapidly, and the last beat or two may fall in instants of relatively low diastolic pressure. If desired, a cardiographic record or a record of

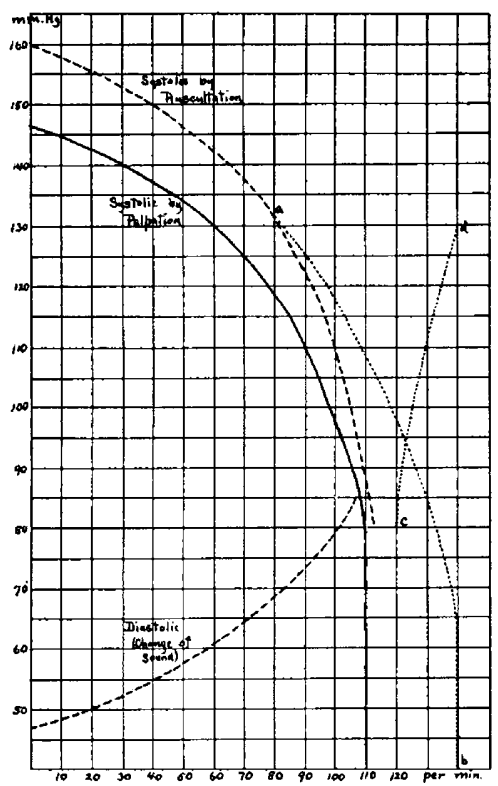

Fig. 4.- Showing the correction of the lower end of the systolic line to represent abortive beats. Hyperthyroidism with auricular fibrillation.

heart sounds could be used for studying the diastolic periods and the probabilities that a certain proportion of the beats would occur during high or low diastolic pressure.

Figure 4, which is from a case of myocarditis with premature contractions (No. 7747), shows that with a cuff pressure of 80 $\mathrm{mm} . \mathrm{Hg}$ all the waves were felt which could be felt with no cuff pressure (110 per minute); and yet each minute there were thirty additional heart beats which could not be felt at the wrist, but which, the sounds were assumed to indicate (second sounds always present), discharged blood into the aorta against a diastolic pressure of 50 to $80 \mathrm{~mm}$. Hg. And, since these abortive beats did not occur in series, it may be assumed that the systolic pressure of the weakest of them 
was probably over $65 \mathrm{~mm}$. $\mathrm{Hg}$. Moreover, it is highly probable that their pressures varied as did those of the larger beats, and that, therefore, if measurable they would be represented by a line approximately in the position of the dotted line $a b$ in Figure 4 ; i. e., a continuation of the auscultatory ${ }^{14}$ systolic line by a more or less symmetrical curve up to the full heart rate (140) and the presumable pressure of the weakest beats $(65 \mathrm{~mm}$. $\mathrm{Hg}$ ). Fortunately, in the region of low pressure this curve is naturally bending so little that the exact location of the point of lowest systolic pressures, which, as explained above, is quite conjectural, makes comparatively little difference in the placement of the line. ${ }^{15}$

14. The fact that systolic readings by palpation are usually somewhat lower than those by auscultation presumably indicates that the auscultatory method is more delicate for determining the pressure of waves which can pass the cuff. And, since in this rough calculation of the pressure of the abortive beats naturally no margin is allowed for failures to demonstrate them, the line representing them should be a continuation of the auscultatory rather than the palpatory systolic line.

15. The line $a b$ representing the systolic pressure of the abortive beats in Figure 4 extends up as high as $130 \mathrm{~mm}$. Hg. A natural question will be why beats with such a pressure cannot be felt in the radial artery with no brachial compression. It is a frequent observation that patients with systolic pressure as low as 50 or $60 \mathrm{~mm}$. Hg (shock, etc.,) have distinctly palpable pulse waves. The answer of course is that the palpability of pulse waves depends not on their absolute pressure, but on the relative increment of pressure, which in turn depends very largely on the volume discharged from the ventricle. That is, with suitable allowances for distensibility of artery walls, interference of surrounding tissues, etc., the case comes under Weber's psychological law (Wagner's Handwörterbuch, Braunschweig, 1846, iii, Abt. 2, p. 511), which is to the effect that for equal increments of sensation, increasing increments of stimulation are necessary. The small pressure increments presumably also prevent many beats with high absolute pressure from giving rise to sounds below the cuff.

The line representing abortive beats in cases other than absolute arrhythmia may obviously have a different type of curve, depending on the conditions. For example, in a case with regular effective beats and regularly recurring abortive premature contractions, the latter might all have about the same pressure, so that they would have to be represented by a nearly horizontal line. In order to locate a rough limit for the pressure of the abortive beats, it would be necessary, as in the case illustrated by Figure 4, to determine the diastolic pressure which they did or did not exceed (presence or absence of second sounds). This could be done approximately by comparing the electrocardiogram or record of heart sounds with an absolute sphygmogram from the case prepared according to the method of Sahli (Diagnostic Methods, 1911, p. 174). The time interval between the abortive beat and the preceding effective beat when marked off on the absolute sphygmogram would indicate, within the limitations of the method, the diastolic pressure at the instant of the abortive beat.

The line $a b$ in Figure 4 may be used according to the directions in Section 2 for constructing the upper end of the diastolic line. Line $c d$ was constructed in this way. It will be realized that this corresponds to the results, which if it were possible, should have been obtained by the use of the fifth phase criterion. This accounts for the fact that the line $c d$ does not join with the main diastolic line of the figure, which, on account of the water-hammer character of the pulse, it was necessary to obtain by the use of the fourth phase criterion. 
The fractional method here described may with propriety be used in many cases other than auricular fibrillation to demonstrate and to measure blood pressure lability; for example, the range of TraubeHering waves or respiratory waves of blood pressure, of sinus arrhythmia, premature contractions, alternating pulse, etc. Figures 5, 6 and 7 are given as examples.

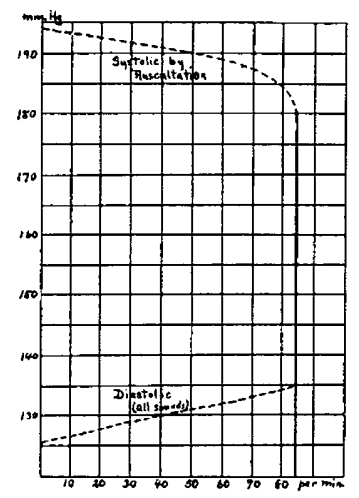

Fig. 5.--Showing a slight amount of pressure lability in an apparently pressure-stable case. Nephritic hypertension.

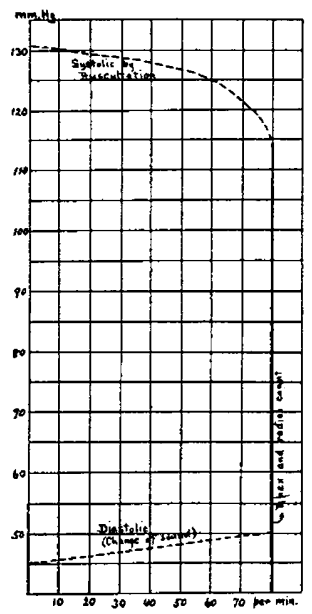

Fig. 6.-Showing a moderate amount of systolic pressure lability due to Cheyne-Stokes respiration. Aortic insufficiency.

Figure 5 is from a case (No. 6220) of nephritic hypertension in which with ordinary examination the pressure seemed stable. The fractional method shows a variation of 5 or $10 \mathrm{~mm}$. $\mathrm{Hg}$ in both systolic and diastolic measurements.

Figure 6 is from a case (No. 6397) of aortic insufficiency in which a moderate amount of systolic variation is attributable to Cheyne- 
Stokes breathing. (Longer counts may be necessary to obtain smooth curves in cases in which the pressure undulations are as slow as in Cheyne-Stokes breathing.)

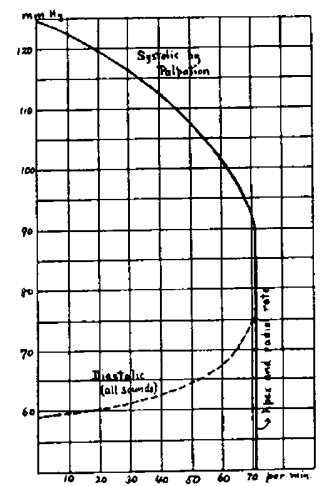

Fig. 7.-Showing much variation of systolic and diastolic pressure due to respiratory arrhythmia.

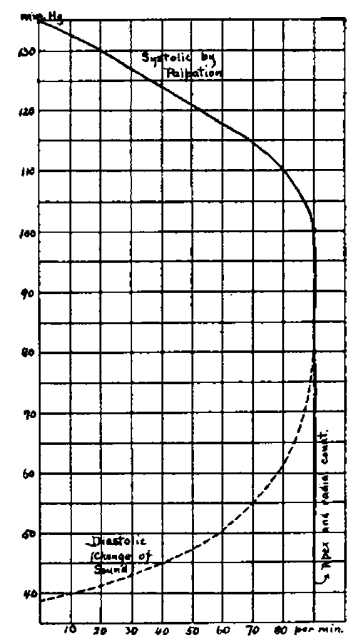

Fig. 8.-Showing a large average pulse pressure as compared with systolic and diastolic pressures in a case of aortic insufficiency with auricular fibrillation.

Figure 7 is from a marked case of respiratory arrhythmia in a boy aged 11 (Case 9680, seen by courtesy of the surgical service). Both systolic and diastolic variability is practically as great as in some of the cases of auricular fibrillation.

\section{DISCUSSION}

The Practical Application of Fractional Blood Pressure Determinations. It is the habit of many clinicians to think of blood pressure as a quantity connected with a patient almost as definite and stable as his 
body weight, or at least as his temperature. Clinical writers who discuss, for example, changes of $5,10,15$, or $20 \mathrm{~mm}$. Hg following certain therapeutic procedures, do not as a rule give sufficient weight to the probable latitude of error in the actual determinations or to the many causes of pressure fluctuations over short time intervals. And even when it is realized that in a case of absolute arrhythmia each pulse wave has a different pressure, the inclination is to combine them into a mean or average figure or to take the pressure of the highest beats, and to attach to these figures the same significance that is carried by the measurements in ordinary cases.

It is hoped that the method here described and the figures used for illustration will help to counteract this tendency, and to emphasize first of all the limitations of human blood pressure determinations ${ }^{16}$ Figures 5 and 6 illustrate, respectively, cases of slight and moderate pressure variability in which in the ordinary run of clinical work some one figure for each case would be reported as the blood pressure. From this as well as from observations on the personal factor of observers, ${ }^{10}$ it would seem necessary that unusual precautions be taken before reliable inferences can be drawn from small differences in blood pressure.

In the frankly pressure-labile cases further precautions are demanded. Both the amount and the kind (shape of the curve) of pressure variation should be taken into consideration. Average systolic and diastolic pressure can always be calculated from the results of the fractional determinations, after correcting the plotted curves when necessary, as described in Sections 2 and 3 . And, with the possible exception of the cases with abortive beats, these averages, based as they are on many observations, may be expected to be as accurate as the usual measurements in normal cases, and probably more so. And in the many instances in which the pressure variation is only moderate and all heart beats produce palpable waves, they undoubtedly bear a fairly close relation to the measurements in pressure-stable cases so far as the usual clinical interpretation of these figures is concerned; i. e., they may be used as indices of hypertension or hypotension, of changes in blood pressure, etc.

The problem is different when many abortive beats are present. If these are included in the calculation, the resulting "average pressure" may have little practical relation to ordinary blood pressure figures. To illustrate: A patient with regular pulse, rate 65 per minute, and systolic pressure 140 , is found to have a regularly recurring premature contraction immediately after each effective heart beat, so that

16. I have reviewed the general question of sources of error in blood pressure determinations in the California State Journal of Medicine, 1914, xii, 92. 
the heart rate is really 130 . The absence of some of the second sounds and the weakness of others belonging to the abortive beats presumably indicates that their systolic pressure is in the neighborhood of the diastolic pressure of the large beats, which is $80 \mathrm{~mm}$. Hg. They cause such small pressure increments, however, that they cannot be felt, and they are negligible so far as the effectiveness of the circulation is concerned. It is obvious that in any consideration of blood flow, vasomotor control, etc., the pressure and the rate of the large beats alone should be taken into account, while the abortive ones would have a separate significance as dissipators of heart energy.

The same is true in cases of premature contractions or auricular fibrillation where the abortive beats are interspersed irregularly. But in these cases there may be every grade of transition between the effective and the abortive beats. The threshold of palpability is a convenient criterion which we have used for distinguishing between the "effective" and the "abortive" groups; and the average pressure of all the beats which can be felt at the wrist may have a somewhat close relationship to the usual measurements in pressure-stable cases. It should be remembered, however, that the distinction at this point is an arbitrary one, and that beats just above the threshold may not have a value for the peripheral circulation proportional to their pressure, while beats just below the threshold may have some value. The highest waves, on the other hand, may not have an efficiency commensurate with their pressure, for the reason that the high pressure is not sustained. Since the ventricle throws out a large volume of blood almost as quickly as a small one, ${ }^{17}$ the rapidity of outflow varies according to the volume of the discharge, and with it also the inertia or "hydraulic ram" action of the blood stream. The latter may run up the instantaneous pressure out of all proportion to the efficiency of the wave. ${ }^{18}$ That is, the same care should here be taken that is necessary in interpreting ordinary blood pressure readings in such cases as aortic insufficiency.

For the reasons given above, the "average blood pressure" of James and Hart, ${ }^{7}$ which includes without distinction the abortive beats, seems to us to be misleading. Certainly for most purposes it is preferable to calculate the average systolic pressure from the palpable beats alone and to give the abortive beats separate consideration.

But if for any cause it is desired to use the general average of both classes of beats, the method of James and Hart would need modification, because it fails to take any account of the pressure of the abortive beats. Since these authors made no use of the method of plotting

17. v. Frey and Krehl: Arch. f. Anat. u. Physiol., 1890, p. 31.

18. Erlanger and Hooker: Johns Hopkins Hosp. Rep., 1904, xii, 145. 
results here described, and did not include diastolic pressure in their determinations, the way was not open for them to state approximately the pressure of those beats; and their method of calculating the average amounted to assigning a zero pressure to all the beats which could not be felt at the wrist. This error accounts for the extraordinarily low pressures recorded in some of their cases. The extended "graph" described in Section 3 to represent these beats makes it possible to apply to them the same averaging method as to the waves which can be felt.

The pulse pressure so far has not been mentioned. It is clear that its average will be the difference between the average systolic and the average diastolic pressure. Also since waves with highest systolic pressure more often follow the longest pauses (and hence as a rule the lowest diastolic pressures), it is probably true that in cases of auricular fibrillation the greatest pulse pressures are approximately measurable by the difference between the highest systolic pressures and the lowest diastolic. Some light on the variation of pulse pressures below this value might be afforded by a study of the diastolic intervals, but the results would be very problematical, and there appears to be no good way to determine the lower limit of pulse pressure. That the average pulse pressure may have some value is indicated by the results in well marked cases of aortic insufficiency with auricular fibrillation. Figure 8 is from a typical case of this kind. The average pulse pressure is considerably greater than the average diastolic pressure. The usefulness of the average pulse pressure, however, as an index of blood flow, etc., will be limited by the considerations mentioned above in regard to systolic pressure.

\section{SUMMARY}

A method is described whereby with the use of the ordinary clinical blood pressure apparatus blood pressure variability may be demonstrated and analyzed. Several types of cardiac arrhythmia are used for illustration. Caution is urged in the interpretation of "average" pressures ; especially a distinction should be made between the effective and the abortive beats.

For helpful suggestions I am indebted to Prof. Joseph Erlanger and to my colleague, Dr. James L. Whitney. 